\title{
COMPETENCIAS Y RESULTADOS DE APRENDIZAJE, UNA VISIÓN DESDE LA CARRERA INGENIERÍA EN SISTEMAS INFORMÁTICOS
}

\author{
Esthela María San Andrés, Universidad Técnica de Manabí ${ }^{1}$ \\ Milagros de la Caridad Rodríguez Andino, Universidad de Camagüey, Cuba²
}

\section{RESUMEN}

El presente trabajo es parte de los resultados parciales de una investigación que se realiza con el fin de perfeccionar el proceso de formación de los Ingenieros en Sistemas Informáticos. Se reflexiona sobre aspectos esenciales relacionados con las competencias y los resultados de aprendizaje, con el propósito de esclarecer ambos conceptos y mostrar de forma práctica como pueden ser formulados los logros de aprendizaje y sus respectivos criterios evaluativos en la materia de Programación II de la cátedra de programación de la UTM.

Palabras claves: Competencias, resultados de aprendizaje, logros de aprendizaje.

\section{ABSTRACT:}

The present work is part of the partial results of an investigation that is carried out with the purpose of perfecting the process of formation of the Engineers in Computer Systems. It is meditated on essential aspects related with the competitions and the learning results, with the purpose of to clarify both concepts and to show in a practical

\footnotetext{
${ }^{1}$ Doctorando (PhD) en Ciencias Pedagógicas en la Universidad de Camagüey (Cuba). Magíster en Informática de Gestión y Nuevas Tecnologías por la Universidad Santa María de Chile (USM), Magíster en Gerencia Educativa por la Universidad Estatal del Sur de Manabí (UNESUM). esanandres@utm.edu.ec, esthelasanandres@hotmail.com.

${ }^{2}$ Doctora en Ciencias de la Educación por la Universidad de la Habana. milagro.rodriguez@reduc.edu.cu, mrodriguezandino@gmail.com. Ha realizado trabajos de investigación y asesoría de tesis en el área de las Ciencias de la Educación y las TIC aplicadas a la enseñanza. Sus publicaciones fundamentales en estos temas han sido publicados en la Base de Datos Scielo y en Latindex.
} 
way as the learning achievements and their respective approaches evaluation can be formulated in the matter of Programming II of the class of programming of the UTM.

\section{Key words: competences, learning, achievement of learning outcomes.}

\section{INTRODUCCIÓN:}

El proceso de evaluación y acreditación es un reto y una oportunidad para avanzar hacia una educación universitaria de mayor calidad, aunque supone un gran esfuerzo por parte de todos, desde las instituciones a los alumnos, pasando indiscutiblemente por los docentes.

Uno de los criterios más importantes del proceso de evaluación señalado en el Modelo General para la Evaluación de Carreras con fines de acreditación (CEAACES, 2012) y que se está implementando en el área de la educación ecuatoriana y latinoamericana con el fin de implementar los principios del Proyecto Tunning para América Latina del cual Ecuador es participe, es el concepto de resultados o logros de aprendizaje.

En la literatura consultada varios autores coinciden en señalar que la evaluación del aprendizaje de los estudiantes no sólo debería estar basada en los conocimientos sobre la materia en cuestión, sino que debe incluir una evaluación basada en las competencias, capacidades y procesos estrechamente relacionadas con el trabajo y las actividades que conducen al progreso del estudiante y a su articulación con los perfiles profesionales definidos.

Esto sitúa, a los profesores, en un escenario distinto, con un enfoque centrado en el estudiante. En este enfoque los resultados de aprendizaje facilitan la tarea del profesor y orientan el aprendizaje del alumno. Anticipan qué serán capaces de hacer 
una vez acabado el aprendizaje, en qué condiciones deberán hacerlo y sugieren criterios que se pueden utilizar para valorar lo aprendido. Asimismo, guían la elección de las estrategias y actividades de aprendizaje, del método de enseñanza y del sistema de evaluación.

En la Universidad Técnica de Manabí, los docentes están inmersos en la aplicación de estas concepciones novedosas al proceso de enseñanza aprendizaje de las diferentes materias, con miras a los futuros procesos de acreditación. Esto ha llevado a las autoras de este trabajo a realizar una investigación en el marco de la carrera de Ingeniería de Sistemas Informáticos, con el propósito de contribuir a una formación adecuada en la cátedra de programación que les permita a los estudiantes un futuro profesional exitoso.

El presente artículo tiene como objetivo mostrar algunas consideraciones de carácter teórico, derivadas del análisis bibliográfico realizado, así como los resultados parciales, referidos a la valoración hecha por las autoras sobre los resultados de aprendizaje, con sus respectivos criterios de evaluación, que deben ser alcanzados en la materia de Programación II por los estudiantes de la carrera de Ingeniería en Sistemas Informáticos (ISI).

\section{METODOLOGÍA:}

En el desarrollo de la presente investigación se ha utilizado el análisis y la síntesis para resumir los fundamentos teóricos esenciales que sirven de sustento a los resultados que se muestran. Además se aplicaron encuestas y entrevistas a profesores y estudiantes de la carrera de ISI con el objetivo de conocer su apreciación sobre la enseñanza aprendizaje de la programación, se muestran algunas de las valoraciones obtenidas. 


\section{DESARROLLO:}

Definiciones actuales señalan que la ISI es la rama de la ingeniería que aplica los fundamentos de la ciencia de la computación, la electrónica y la ingeniería de software, para el desarrollo de soluciones integrales de cómputo y comunicaciones, capaces de procesar información de manera automática.

En general está señalado en la bibliografía actual que los Ingenieros en Sistemas informáticos están involucrados en los sistemas informáticos de grandes, computadoras de escritorio, televisores de alta definición, así como ordenadores integrados para teléfonos móviles, videojuegos, sistemas de realidad virtual, electrodomésticos inteligentes e implantes médicos, por lo que estos ingenieros se deben preparar para analizar, diseñar, desarrollar y fabricar todo tipo de productos digitales y los sistemas que incluyen tanto hardware como software. Asimismo deben planificar, diseñar, monitorear, optimizar y administrar sistemas informáticos complejos, para lo cual el desarrollo de programas de software es un elemento importante.

Al respecto, la revisión hecha por las autoras a la concepción de la Ingeniería de Sistemas Informáticos a nivel nacional en la Universidad Autónoma de Quito, Universidad San Francisco de Quito, Universidad Central del Ecuador, Escuela Superior Politécnica del Ecuador ESPE, Escuela de Ingeniería de la Universidad de Azuay, Universidad Laica Eloy Alfaro de Manabí (ULEAM), Universidad Técnica de Manabí UTM y a nivel internacional (Universidad Nicaragüense de Ciencia y Tecnología, Escuela de Ingeniería de San Salvador, Universidad Iberoamericana, Universidad Mayor San Andrés Bolivia, Universidad Nacional de Colombia, , 
Universidad de los Andes, Universidad Nacional Autónoma de México, Tecnológico de Monterrey, Universidad de Panamá, Universidad Politécnica de Madrid, Universidad Complutense de Madrid, Universidad de Chile; Universidad Tecnológica Nacional, Universidad de Buenos Aires; Universidad Abierta Interamericana de Argentina; Universidad Nacional Experimental de Tachira Venezuela; Florida International University, Harvard School of Engineering and Applied Sciences; Universidad Mesoamericana, Universidad Rafael Landivar de Guatemala), posibilita establecer las siguientes características en su perfil profesional:

- Formado para la investigación y el avance científico- tecnológico

- Preparado para planificar, diseñar, implantar, conceptualizar, analizar, usar, evaluar y dar soporte lógico a las empresas públicas y privadas.

- Planifica, diseña, dimensiona y evalúa los requerimientos del soporte físico utilizado en el tratamiento del proceso automático de datos en empresas.

- Participa en los procesos de auditoría y fiscalización informática

- Realiza labores de consultoría en informática a nivel superior y medio.

- Desempeña funciones de dirección, de asesoría o colaboración en informática y computación.

- Forma parte de grupos multidisciplinarios de investigación.

- Establece y aplica las pruebas de calidad

- Gerencia empresas de desarrollo de productos de software.

En un estudio realizado de los resultados de la reunión de Bruselas de noviembre del 2012 en el Área Informática del Proyecto Tuning América Latina, en su 
proyección 2012-2013, presentado por el coordinador de esta área (Contreras, J, 2012) se observa que los especialistas que trabajan en esta propuesta señalan que: los perfiles profesionales y competencias que se definan deben estar basados en la esencialidad que posibiliten el constante cambio, adaptabilidad del entorno y un aumento en la formalidad de la profesión y también en la especificidad que posibiliten la asimilación temprana de áreas de aplicación particulares.

El proyecto ha propuesto 13 competencias profesionales para el área de Informática, Sistemas y Computación con orientación internacional. En la Tabla 1 se muestran cuales son estas competencias.

El criterio de las autoras de esta investigación es que las diferentes carreras de este perfil deben revisar esta propuesta y perfeccionar el perfil profesional y las competencias de las carreras vinculadas con el área informática de manera tal que se ajusten a las nuevas exigencias y demandas a nivel mundial y que emanan de los análisis del Proyecto Tuning para América Latina, con el fin de buscar coherencia en la formación de profesionales de las distintas titulaciones que corresponden a esta área, en los diferentes países respetando los aspectos de carácter específico que puedan emanar de los distintos contextos, lo que permitirá prever a los estudiantes de oportunidades para el desarrollo de trabajos en contextos internacionales.

Una valoración importante para lograr este propósito es comprender el concepto de competencia profesional o competencia para una profesión específica, esta es tratada por varios autores entre ellos, Oliver et al. (2008), a partir de la definición dada por Chambers (1994) distingue que la competencia para una profesión específica es el comportamiento esperado al comenzar un profesional de forma 
independiente. Este comportamiento incorpora la comprensión, destrezas y valores en una respuesta integrada en relación a una amplia variedad de circunstancias enfrentadas en la práctica profesional en términos generales, (Oliver et al., 2008). Por otra parte (Forgas J.A., 2001) señala que la competencia profesional es el resultado de la integración, esencial y generalizada de un complejo conjunto de conocimientos, habilidades y valores profesionales, que se manifiesta a través de un desempeño profesional eficiente en la solución de los problemas de su profesión, pudiendo incluso resolver aquellos no predeterminados.

Para la formación y desarrollo de una competencia se necesita la descripción de las competencias en términos de resultados o de logros de aprendizaje.

Es necesario definir, lo más exactamente posible, cada una de las competencias de un perfil de egreso, de un programa, de manera que queden bien delimitadas. Por ello para cada competencia debe establecerse:

- Los resultados de aprendizaje asociados a la demostración de la competencia.

- Los criterios de evaluación que van a permitir medir el éxito de la formación.

- El medio en el cual se desarrollaría la evaluación

Los resultados de aprendizaje apoyan a las competencias, son más detallados y forman la base tanto del aprendizaje como de la apreciación. Los resultados de aprendizaje son formulados para indicar lo que los estudiantes deben saber, lo que los estudiantes deben comprender, y lo que los estudiantes deben ser capaces de hacer y cuán bien, utilizando el lenguaje y el contexto que indican el nivel en el cual van a ser apreciados. (Oliver et al., 2008). 


\section{Resultados de Aprendizaje:}

Las tendencias internacionales en educación muestran un cambio del enfoque "centrado en el profesor" a un enfoque "centrado en el estudiante". Este modelo alternativo se centra en lo que los estudiantes deben ser capaces de hacer al término del módulo o programa. De ahí que este enfoque se refiere comúnmente a un enfoque basado en resultados o logros del aprendizaje.

El empleo de resultados de aprendizaje y competencias es necesario para hacer que los programas de estudio y sus unidades o módulos de curso estén centrados en el estudiante y se orienten a outputs o sea hacia las salidas. Este planteamiento requiere que los conocimientos y habilidades más importantes que un estudiante tenga que adquirir durante el proceso de aprendizaje determinen los contenidos del programa de estudios. Los resultados del aprendizaje y las competencias se centran en los requerimientos de la disciplina y la sociedad en términos de preparación para el mercado de trabajo y la ciudadanía.

La definición de resultados del aprendizaje acordada en el Proyecto Tuning para América Latina (Proyecto Tuning, 2007, 323) es la siguiente: Formulaciones que el estudiante debe conocer, entender o ser capaz de demostrar una vez concluido el proceso de aprendizaje. Los resultados del aprendizaje deben estar acompañados de criterios de evaluación adecuados que pueden ser empleados para juzgar si se han conseguido los resultados previstos.

Otra definición más actual es la emitida por CEAACES (2011) donde se señala que los resultados o logros del aprendizaje describen el aprendizaje en términos de comportamientos, conocimientos y actitudes en niveles de cumplimiento 
específicos, es decir, lo que el alumno será capaz de conocer, comprender y de hacer al término de un proceso de aprendizaje y/o de sus estudios de la carrera.

El proceso de aprendizaje puede ser, por ejemplo, una clase, un módulo o un programa completo.

La distinción entre los resultados del aprendizaje y la competencia se hace claro en la recomendación de $\mathrm{EQF}^{3}$, aquí los resultados de aprendizaje se definen como "lo que un aprendiz reconoce y es capaz de hacer al finalizar un proceso de aprendizaje..." mientras que la competencia significa "la capacidad demostrada para utilizar conocimientos, habilidades y capacidades personales, sociales $\mathrm{y} / \mathrm{o}$ metodológicas, en el estudio o situaciones de trabajo y en el desarrollo profesional y personal...", competencias, por tanto, son las características que una persona muestran en acción.

\section{La redacción de los resultados de aprendizaje:}

Hay una gran cantidad de información en la literatura especializada referida a lo que se considera el mejor procedimiento para redactar resultados de aprendizaje (Bingham, 1999; Fry et al., 2000; Jenkins y Unwin, 2001; Moon, 2002). En términos generales, cuando se redactan resultados de aprendizaje, es útil centrarse en lo que se espera de los estudiantes que sean capaces de hacer o demostrar al término del módulo o del programa.

Es importante que los resultados de aprendizaje se expresen en términos simples y no ambiguos, de manera que los estudiantes, profesores, empleadores y examinadores externos los puedan entender en forma clara.

\footnotetext{
${ }^{3}$ Recommendation of the European Parliament and of the Council on the establishment of the European Qualifications Framework for lifelong Learning $\underline{h t t p: / / e u r-l e x . e u r o p a . e u /}$
} 
Los resultados de aprendizaje especifican por lo general el aprendizaje esencial para un módulo. Por lo tanto, cuando se redactan, se ha acordado especificar el mínimo aceptable que permita al estudiante aprobar el módulo.

La Taxonomía de Bloom (Bloom, 1956), como señalan varios autores (Anderson, 2001; Kennedy, 2007) es una de las ayudas más útiles para redactar resultados de aprendizaje bien hechos. Esta taxonomía proporciona una lista de verbos confeccionada y es por ende una "herramienta" útil que provee la terminología para redactar resultados de aprendizaje.

Según Bloom, se puede distinguir tres tipos principales de resultados o logros del aprendizaje: en el dominio cognitivo (saber), en el dominio de las aptitudes (saber hacer) y en el dominio de las actitudes (saber ser). Para cada uno de ellos se dispone de una serie de verbos que describen los distintos niveles requeridos por la formación profesional, (Figura 1).

Neary (2002) con respecto a la enseñanza por resultados, señala que es un reto para el profesor seleccionar los resultados de aprendizaje apropiados que conduzcan a adquirir las competencias, especificar los indicadores evaluativos y desarrollar un sistema funcional de entrega.

Algunas recomendaciones brindadas por Kennedy, 2007 para la Redacción de resultados de aprendizaje son:

- Comience cada resultado de aprendizaje con un verbo de acción, seguido por el complemento del verbo y por una frase que le provea contexto.

- Utilice sólo un verbo para cada resultado de aprendizaje. 
- Evite términos vagos como saber, comprender, aprender, estar familiarizado con, estar expuesto a, estar consciente de.

- Asegúrese que los resultados de aprendizaje de la asignatura o módulo están en función de las competencias genéricas y específicas derivadas del perfil de titulación.

- Pregúntese si los resultados de aprendizaje que está redactando son observables y medibles.

- Asegúrese que podrá evaluar los resultados de aprendizaje. Pregúntese cómo lo hará.

- Pregúntese si es posible lograr los resultados de aprendizaje dentro del tiempo y los recursos disponibles.

- Trabaje redactando entre 4 y 6 resultados de aprendizaje por asignatura o módulo

Los resultados de aprendizaje escritos para diferentes disciplinas pueden diferir en sus componentes debido a las estructuras de conocimiento. La naturaleza de la materia de estudio, determinará el nivel y las palabras adicionales que indiquen la profundidad del conocimiento. La profundidad o el contexto del conocimiento indicarán el nivel con que se debe adquirir ese resultado o logro de aprendizaje en la asignatura.

\section{RESULTADOS Y DISCUSIÓN:}

La realización de un diagnóstico para comprobar el nivel de preparación que tienen los estudiantes de $8 \mathrm{vo}, 9$ no y $10 \mathrm{mo}$ nivel de la carrera de Ingeniería en Sistemas 
Informáticos de la UTM, ha permitido conocer que existen falencias relacionadas con la enseñanza aprendizaje de la programación.

Entre las principales dificultades están:

- De los estudiantes encuestados un $76 \%$ considera que a veces los conocimientos que están adquiriendo son los necesarios para realizar con éxito la solución de los problemas que demandan las exigencias sociales a los Ingenieros en Sistemas Informáticos. (Observar Tabla 2 y Gráfico 1).

- Un $66 \%$ considera que a veces los procedimientos y métodos que se utilizan en las clases de programación son los adecuados para darle solución a los problemas que debe enfrentar en la profesión (Observar Tabla 3 y Gráfico 2).

- El $55 \%$ y $22 \%$ consideran que a veces o casi nunca en las clases de laboratorio se discuten las variantes de los ejercicios de Programación orientados por los docentes (Observar Tabla 4 y Gráfico 3).

Estos resultados señalan que es necesario repensar el trabajo que se realiza, nos indica que se necesita un perfeccionamiento de manera que la metodología utilizada por el docente en las asignaturas conlleve a la obtención de los resultados de aprendizaje declarados para la carrera, según lo establecido por $(\mathrm{ABET})^{4}$, los cuales son:

a) Capacidad de realizar análisis, síntesis y aplicación de las matemáticas y ciencias básicas en la solución de problemas de ingeniería en sistemas informáticos.

\footnotetext{
${ }^{4}$ ABET, Organización reconocida en los Estados Unidos que acredita los programas de Ingeniería, Tecnología, Computación y Ciencias Aplicadas de los Institutos de Educación Superior y Universidades.
} 
b) Capacidad de planificar, diseñar, conducir e interpretar resultados de experimentos orientados a la informática.

c) La capacidad de diseñar sistemas, procesos, modelos y componentes informáticos que cumplan los estándares nacionales o internacionales, tomando en cuenta las limitaciones económicas, ambientales, sociales, políticas, de salud y seguridad del entorno, y cumpliendo satisfactoriamente con las especificaciones y restricciones existentes 0 indicadas por los interesados o por los criterios de sostenibilidad.

d) Capacidad para funcionar como parte de un equipo de profesionales de distintas áreas del conocimiento, demostrando una efectiva cooperación, comunicación, con habilidades para resolver conflictos y contribuyendo proactivamente en la propuesta de líneas estratégicas desde el punto de vista informático, para la solución de problemas.

e) Capacidad para identificar, formular, evaluar y resolver técnicamente problemas de ingeniería planteados de acuerdo a las necesidades del medio.

f) Capacidad para comprender, reconocer y aplicar valores y códigos de ética profesional, que le permitan desenvolverse sin perjudicar a sus clientes y contribuyendo al desarrollo de la sociedad.

g) Habilidad para presentar efectivamente, ideas, proyectos, informes de investigaciones, documentos de trabajo de manera escrita, oral y digital, utilizando las herramientas de las nuevas tecnologías de la información.

h) Habilidad y capacidad para comprender el impacto de las soluciones informáticas a la realidad local, nacional e internacional en un contexto económico global, ambiental y social. 
i) Habilidad y aptitud para ser un profesional con el compromiso del aprendizaje continuo, con capacidad para reconocer las oportunidades para mejorar en su campo profesional

j) Habilidad para identificar temas y problemas de actualidad con respecto al entorno local, regional y global, con el fin de relacionarlos con propuestas de soluciones creativas y eficientes.

k) Capacidad y destreza para utilizar técnicas, habilidades y herramientas en el desarrollo de software y hardware para implementar soluciones a problemas de su profesión.

Sobre la base de estos resultados y de las competencias profesionales o específicas del perfil del graduado declaradas en el Proyecto Turning para América Latina se ha realizado un análisis de las asignaturas dedicadas a las materias de programación. En este trabajo se mostrará parte de los resultados de aprendizajes relacionados con la cátedra Programación II, definiendo la competencia y resultado de aprendizaje del perfil descriptivo de la carrera que se relaciona con la asignatura, los resultados de aprendizaje que deben alcanzarse en la asignatura, los criterios para su evaluación, los niveles ha alcanzar por cada resultados de aprendizaje y su ponderación. (Observar Tabla 5)

\section{CONCLUSIONES:}

- $\quad$ La adquisición de competencias es un largo proceso, no se evidencia en la acumulación de créditos, sino en la demostración de una capacidad de desempeño en situaciones específicas.

- Los resultados de aprendizaje juegan un rol importante al asegurar transparencia en las capacidades y en sus conceptos, valores y prácticas. 
- Los resultados del aprendizaje están vinculados a los niveles correspondientes y generalmente deben ser valorables deben escribirse en términos de cómo se representa el aprendizaje

- $\quad$ El trabajo del estudiante, los métodos de enseñanza, las competencias y los resultados del aprendizaje están claramente relacionados entre sí.

\section{TABLAS Y LEYENDAS DE LAS FIGURAS}

\section{COMPETENCIAS EN EL ÁREA DE INFORMÁTICA}

1 Aplicar el conocimiento de ciencias de la computación, de tecnologías de la info rmación, y de las organizaciones, para desarrollar soluciones informáticas.

2 Concebir, diseñar, desarrollar y operar soluciones informáticas basándose en pri ncipios de ingeniería y estándares de calidad.

3 Aplicar el enfoque sistémico en el análisis y resolución de problemas.

4 Aplicar fundamentos matemáticos, principios algorítmicos y teorías de Ciencias de la Computación en la modelación y diseño de soluciones informáticas.

5 Desempeñar diferentes roles en proyectos informáticos, en contextos multidiscip linarios y multiculturales, tanto locales como globalizados.

6 Aplicar su conocimiento en forma independiente e innovadora en la búsqueda de soluciones informáticas, con responsabilidad y compromiso social.

7 Identificar oportunidades para mejorar el desempeño de las organizaciones a tra vés del uso eficiente y eficaz de soluciones informáticas

8 Liderar procesos de incorporación, adaptación, transferencia y producción de $\mathrm{s}$ oluciones

informáticas para apoyar los objetivos estratégicos de las organizaciones.

9 Aplicar estándares de calidad en el desarrollo y evaluación de soluciones informáticas.

10 Comprender y aplicar los conceptos éticos, legales, económicos y financieros pa ra la toma de decisiones y para la gestión de proyectos informáticos.

11 Liderar emprendimientos en la creación de productos y servicios vinculados con la informática.

12 Aplicar metodologías de investigación en la búsqueda, fundamentación y elabor ación de soluciones informáticas.

13 Asimilar los cambios tecnológicos y sociales emergentes.

Tabla1: Competencias en el área de informática definidas en el marco del Proyecto Tuning para América Latina en Bruselas Nov. 2012 


\begin{tabular}{|r|l|r|r|r|}
\hline$\#$ & Alternativas & \multicolumn{1}{|c|}{$\mathbf{f}$} & \multicolumn{1}{|c|}{$\mathbf{f r}$} & \multicolumn{1}{|c|}{$\%$} \\
\hline 5 & Siempre & 14 & 0,08 & 7,82 \\
\hline 4 & Casi Siempre & 26 & 0,15 & 14,53 \\
\hline 3 & A veces & 137 & 0,77 & 76,54 \\
\hline 2 & Casi Nunca & 2 & 0,01 & 1,12 \\
\hline 1 & Nunca & 0 & 0,00 & 0,00 \\
\hline & & 179 & 1,00 & $\begin{array}{r}\text { Estoy adquiriendo los conocimientos necesarios } \\
\text { para realizar con éxito la solución de problemas } \\
\text { que demandan las exigencias sociales a los } \\
\text { Ingenieros en sistemas Informáticos }\end{array}$ \\
\hline
\end{tabular}

\begin{tabular}{|c|c|c|c|c|c|}
\hline$\#$ & Alternativas & $f$ & $\mathrm{fr}$ & $\%$ & \multirow{4}{*}{$\begin{array}{l}\text { Los procedimientos y métodos utilizados en } \\
\text { las clases de program ación son adecuados } \\
\text { para la solución de los problemas que debe } \\
\text { enfrentar en la profesión } \\
\end{array}$} \\
\hline 5 & Siempre & 12 & 0,07 & 6,70 & \\
\hline 4 & Casi Siempre & 43 & 0,24 & 24,02 & \\
\hline 3 & A veces & 119 & 0,66 & 66,48 & \\
\hline 2 & Casi Nunca & 4 & 0,02 & 2,23 & D Siempre \\
\hline 1 & Nunca & 1 & 0,01 & 0,56 & $\square$ Aveces \\
\hline & TOTAL........ & 179 & 1,00 & $\begin{array}{r}100,0 \\
0 \\
\end{array}$ & $\begin{array}{l}\text { Casi Nunca } \\
\text { Q Nunca }\end{array}$ \\
\hline
\end{tabular}

\begin{tabular}{|r|l|r|r|r|}
\hline$\#$ & Alternativas & f & fr & \multicolumn{1}{|c|}{$\%$} \\
\hline 5 & Siempre & 27 & 0.15 & 15.08 \\
\hline 4 & Casi Siempre & 12 & 0.07 & 6.70 \\
\hline 3 & A veces & 99 & 0.55 & 55.31 \\
\hline 2 & Casi Nunca & 40 & 0.22 & 22.35 \\
\hline 1 & Nunca & 1 & 0.01 & 0.56 \\
\hline & TOTAL....... & 179 & 1.00 & 100.00 \\
\hline \multicolumn{7}{|c|}{$\begin{array}{l}\text { Se Discuten variantes de respuestas de los ejercicios } \\
\text { de Programación orientados por los docentes }\end{array}$} \\
\hline $\begin{array}{l}\text { Tabla 4 y Gráfico 3: En las clases prácticas de las cátedras de programación se discuten las } \\
\text { variantes de respuestas de los ejercicios que orienta el docente? }\end{array}$
\end{tabular}




\section{DEL PERFIL DE EGRESO:}

COMPETENCIA: Concibe, diseña, desarrolla y opera soluciones informáticas basándose en principios de ingeniería y estándares de calidad.

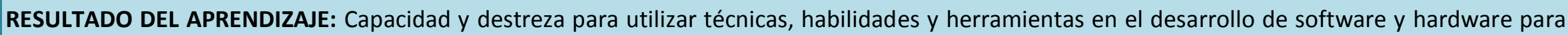
implementar soluciones a problemas de su profesión.

COMPETENCIA DE LA ASIGNATURA : Resuelve problemas de programación usando un nivel adecuado de abstracción que le permita diseñar e implementar software de manera efectiva usando el paradigma de programación Orientada a Objetos.

\begin{tabular}{|c|c|c|c|c|}
\hline \multirow{2}{*}{$\begin{array}{l}\text { RESULTADOS DEL } \\
\text { APRENDIZAJE DE LA } \\
\text { ASIGNATURA }\end{array}$} & \multicolumn{4}{|c|}{ METODOLOGÍA DE EVALUACIÓN DE LOS RESULTADOS DE APRENDIZAJE } \\
\hline & $\begin{array}{l}\text { FORMAS DE EVIDENCIARLOS } \\
\text { (Apreciación) }\end{array}$ & CRITERIOS & $\begin{array}{l}\text { NIVELES DEL } \\
\text { RESULTADO DE } \\
\text { PRENDIZAJE }\end{array}$ & PONDERACIÓN \\
\hline $\begin{array}{l}\text { UNIDAD: } 1 \\
\text { Distinguir entre la } \\
\text { Programación } \\
\text { Estructurada clásica y la } \\
\text { Programación Orientada a } \\
\text { Objetos. }\end{array}$ & $\begin{array}{l}\text { - Pruebas escritas, orales o en el computador } \\
\text { - Exposiciones } \\
\text { - Ensayos } \\
\text { - Trabajos Investigativos (Informes) } \\
\text { - Actividades en grupos pequeños/tutorías } \\
\text { - docentes. } \\
\text { - Votos } \\
\text { - Videos }\end{array}$ & $\begin{array}{l}\text { - Conceptualizará a través de un ensayo los términos } \\
\text { fundamentales utilizados en la programación orientada a } \\
\text { objetos. } \\
\text { - Conceptualizará a través de un ensayo al menos } 4 \text { términos } \\
\text { fundamentales utilizados en la programación orientada a } \\
\text { objetos. } \\
\text { - Conceptualizará a través de un ensayo al menos } 3 \text { términos } \\
\text { fundamentales utilizados en la programación orientada a } \\
\text { objetos. }\end{array}$ & $\begin{array}{l}\text { Medio } \\
\text { Básico }\end{array}$ & $\begin{array}{c}71 \%-85 \% \\
70 \%\end{array}$ \\
\hline $\begin{array}{l}\text { UNIDAD: } 2 \\
\text { Construir funciones y } \\
\text { miembros adecuados en el } \\
\text { desarrollo de la estructura } \\
\text { de una clase. }\end{array}$ & $\begin{array}{l}\text { - Ensayos } \\
\text { - Actividades en grupos pequeños/tutorías } \\
\text { docentes. } \\
\text { - Trabajos Investigativos (Informes) } \\
\text { - Fotos } \\
\text { - Pruebas en el computador }\end{array}$ & $\begin{array}{l}\text { - Construirá clases que permitan implementar } 3 \text { objetos que } \\
\text { puedan comunicarse entre sí por medio de mensajes } \\
\text { parametrizados. } \\
\text { - Construirá clases que permitan implementar } 2 \text { objetos que } \\
\text { puedan comunicarse entre sí por medio de mensajes } \\
\text { parametrizados. } \\
\text { - Construirá clases que permitan implementar } 1 \text { objeto. }\end{array}$ & $\begin{array}{l}\text { Alto } \\
\text { Medio } \\
\text { Básico }\end{array}$ & $\begin{array}{c}86 \%-100 \% \\
71 \%-85 \% \\
70 \%\end{array}$ \\
\hline $\begin{array}{l}\text { UNIDAD: } 3 \\
\text { Definir función } \\
\text { constructora y destructora } \\
\text { por defecto o con } \\
\text { parámetros. }\end{array}$ & $\begin{array}{l}\text { - Ensayos } \\
\text { - Actividades en grupos pequeños/tutorías } \\
\text { docentes. } \\
\text { - Trabajos Investigativos (Informes) } \\
\text { - Fotos } \\
\text { - Pruebas en el computador. }\end{array}$ & $\begin{array}{l}\text { - Creará programas con la ayuda del computador en el lenguaje de } \\
\text { programación indicado por el docente, empleando los diferentes } \\
\text { tipos de constructores y destructores por defecto y por } \\
\text { parámetros. } \\
\text { - Creará programas con la ayuda del computador en el lenguaje de } \\
\text { programación indicado por el docente empleando los tipos de } \\
\text { constructores y destructores por parámetros. } \\
\text { - Creará programas con la ayuda del computador en el lenguaje de } \\
\text { programación indicado por el docente empleando los tipos de } \\
\text { constructores por defecto. }\end{array}$ & $\begin{array}{l}\text { Alto } \\
\text { Medio } \\
\text { Básico }\end{array}$ & $\begin{array}{c}86 \%-100 \% \\
71 \%-85 \% \\
70 \%\end{array}$ \\
\hline
\end{tabular}

Tabla 5: Resultados de aprendizajes en la cátedra Programación II, con su respectiva competencia y resultado de aprendizaje del perfil descriptivo relacionada con la asignatura. 


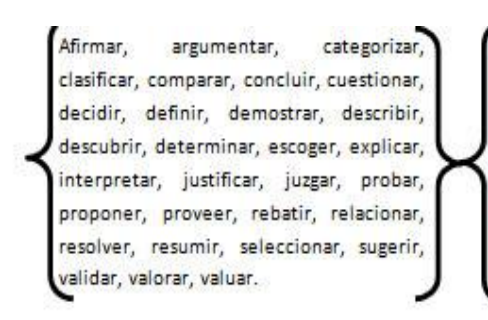

$\left\{\begin{array}{l}\text { Bosquejar, clasificar, categorizar, } \\ \text { combinar, componer, crear, construir, } \\ \text { decir, deducir, desarrollar, diseñar, } \\ \text { derivar, escribir, establecer, explicar, } \\ \text { estructurar, extraer, formular, } \\ \text { generalizar, idear, integrar, inventar, } \\ \text { inventariar, modificar, hacer, dirigir, } \\ \text { organizar, originar, planificar, } \\ \text { producir, proponer, reconstruir, etc. }\end{array}\right\}$

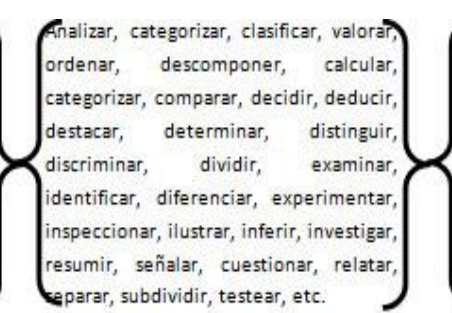

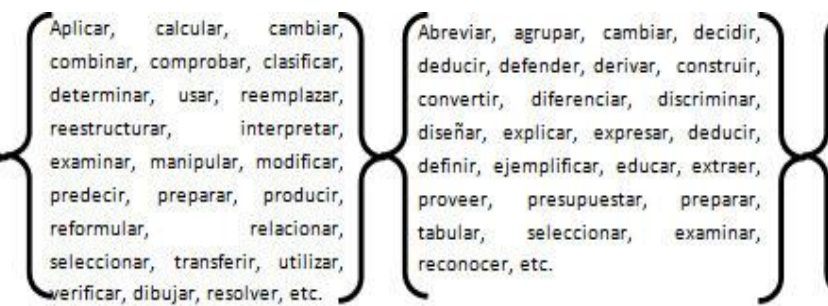
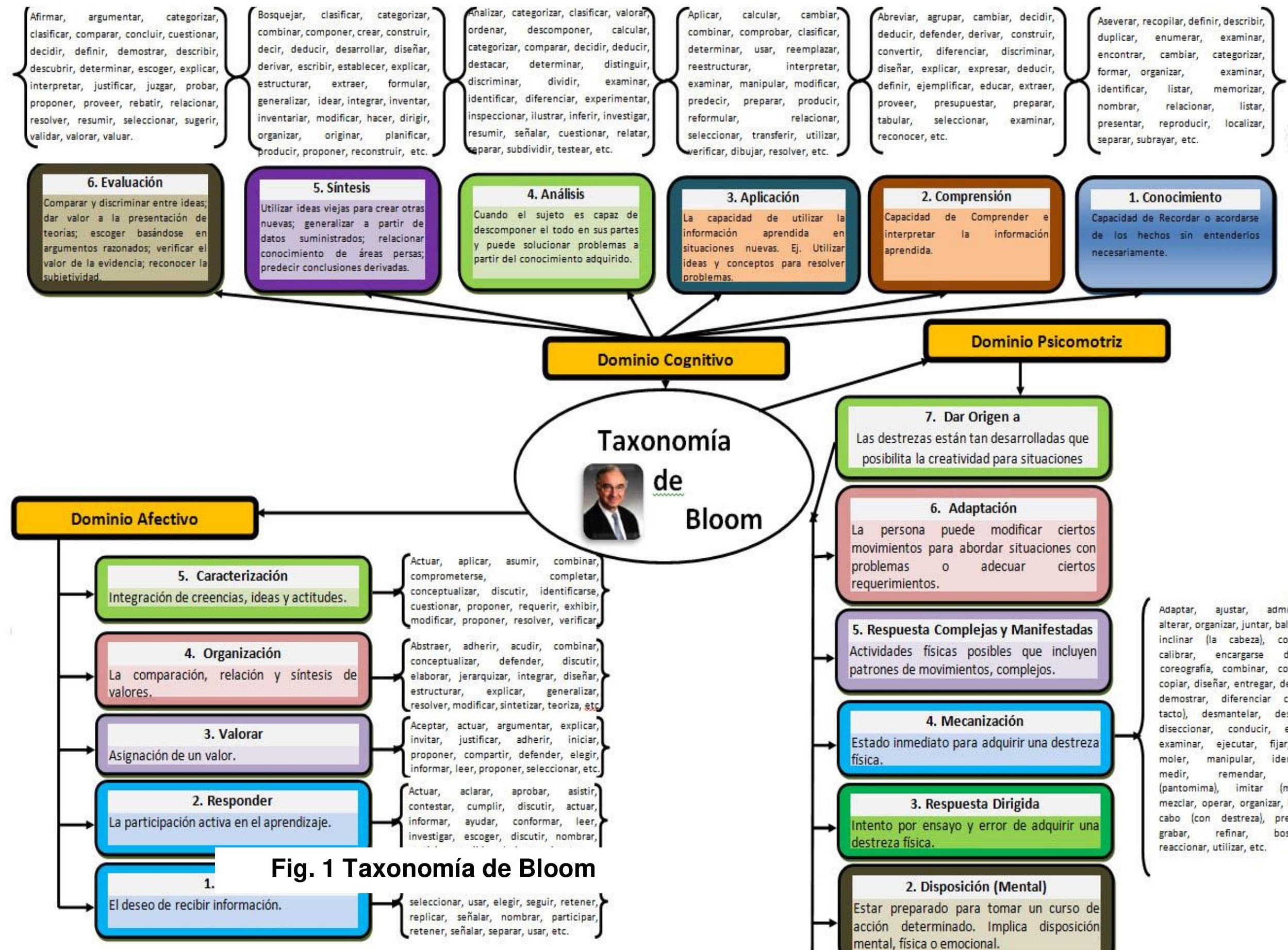

7. Dar Origen a

Las destrezas están tan desarrolladas que

posibilita la creatividad para situaciones

La persona puede modificar ciertos movimientos para abordar situaciones con problemas 0 adecuar ciertos requerimientos.

5. Respuesta Complejas y Manifestadas Actividades fisicas posibles que incluyen patrones de movimientos, complejos.

\section{Mecanización} stado inmediato para adquirir una destreza

3. Respuesta Dirigida intento por ensayo y error de adquirir una destreza fisica.

2. Disposición (Mental)

Estar preparado para tomar un curso de acción determinado. Implica disposición mental, fisica o emociona.

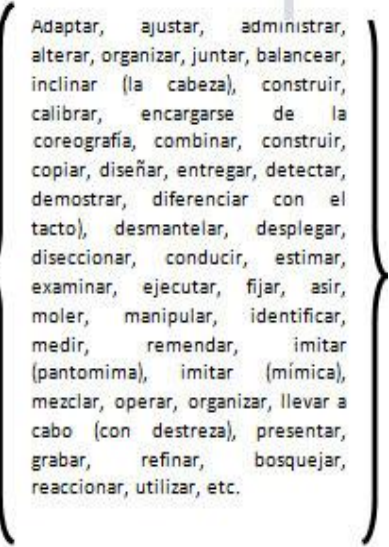
Adaptar, ajustar, administrar,
alterar, organizar, juntar, balancer,
inclinar (la cabeza), construir,
calibrar, encargarse de la
coreografia, combinar, construir,
copiar, diseñar, entregar, detectar,
demostrar, diferenciar con el
tacto), desmantelar, desplegar,
diseccionar, conducir, estimar,
examinar, ejecutar, fijar, asir,
moler, manipular, identificar,
medir, remendar, imitar
(pantomima), imitar (mimica),
mezclar, operar, organizar, llevar a
cabo (con destreza), presentar,
grabar, refinar, bosquejar,
reaccionar, utilizar, etc.
4 


\section{REFERENCIAS BIBLIOGRÁFICAS:}

Adam,S. (2004), Using Learning Outcomes: A consideration of the nature, role, application and implications for European education of employing learning outcomes at the local, national and international levels. Report on United Kingdom Bologna Seminar, July 2004, Herriot-Watt University.

Allan, J. (1996), Learning outcomes in higher education, Studies in Higher Education, 21 (10)pp. $93-108$.

Anderson, L.W., \& Krathwohl, D. (Eds.) (2001), A Taxonomy for Learning, Teaching and Assessing: A Revision of Bloom's Taxonomy of Educational Objectives. New York: Longman.

Bingham, J. (1999), Guide to Developing Learning Outcomes. The Learning and Teaching Institute Sheffield Hallam University, Sheffield: Sheffield Halllam University.

Bloom, B.S. (1956), Taxonomy of Educational Objectives, Book 1 Cognitive Domain. Longman Publishing.

CEAACES (2011). Modelo general para la evaluación de carreras con fines de acreditación. Quito.

Council of Europe (2002), Seminar on Recognition Issues in the Bologna Process, Lisbon, April 2002. (http://www.coe.int)

Contreras, J. (2012), Área Informática. Tuning América Latina: Innovación Educativa y Social. Reunión de Bruselas. Disponible en: http://www.tuningal.org/es/publicaciones/cat view/44-presentaciones-reunionbruselas-19-22-nov-2012. Consultado: Enero 2013.

ECTS Users' Guide (2005), Brussels: Directorate-General for Education and Culture, Available online at:

http://ec.europa.eu/education/programmes/socrates/ects/doc/guide en.pdf

EQF, (2011).Using Learning outcomes. European Union. Disponible en: http://ec.europa.eu/education/lifelong-learning-policy/doc/egf/note4 en.pdf.

Consultado: Febrero 2013.

Ferris, T. and Aziz, S. (2005), A psychomotor skills extension to Bloom's Taxonomy of Education Objectives for engineering education. Exploring Innovation in Education and Research, March 2005.

Fry, H., Ketteridge, S., Marshall (2000), A Handbook for Teaching and Learning in Higher Education. London: Kogan Page. 
FORGAS, J. 2001. Modelo para la formación profesional en la Educación Técnica y Profesional sobre la base de Competencias Profesionales en la rama de Mecánica. Conferencia. Jamaica.

Gosling,D. and Moon, J. (2001), How to use Learning Outcomes and Assessment Criteria. London: SEEC Office.

Harrow, A. (1972), A taxonomy of the psychomotor domain - a guide for developing behavioral objectives. New York: David McKay.

Jenkins, A. \& Unwin, D. (2001), How to write learning outcomes. Disponible en: www.ncgia.ucsb.edu/eduacation/curricula/giscc/units/format/outcomes.html

Kennedy, D. (2007). Redactar y utilizar resultados de aprendizaje. Disponible en: http://www.uctemuco.cl/cedid/archivos/apoyo/new resultados de aprendizaje 01 dkennedy.pdf . Consultado: Febrero 2013.

Krathwohl, David, R. (2002), A Revision of Bloom's Taxonomy: An Overview. Theory into Practice, 41 (4).

Moon, J. (2002), The Module and Programme Development Handbook. London: Kogan Page Limited.

Tuning, AL. (2007). Reflexiones y perspectivas de la Educación Superior en America Latina, Informe Final. Disponible en: http://www.tuning.unideusto.org/tuningal. Consultado: Enero 2013.

Simpson, E. (1972), The classification of educational objectives in the psychomotor domain: The psychomotor domain. Vol.3. Washington DC: Gryphon House.

Yániz, C. y Villardón, L. (2006). Planificar desde competencias para promover el aprendizaje. El reto de la sociedad del conocimiento para el profesorado universitario. Bilbao: ICE de la UD. Cuadernos monográficos del ICE, núm. 12. 